$16 \mid 2020$

Les marges créatrices : intellectuel.le.s afrodescendant.e.s et indigènes auX Amériques, XIX-XXe siècle

\title{
Le miroir haïtien de Ben Fountain
}

Ben Fountain's Haitian Mirror

Espelho haitiano de Ben Fountain

\section{Françoise Palleau-Papin}

\section{OpenEdition}

Journals

Édition électronique

URL : http://journals.openedition.org/ideas/8698

DOI : $10.4000 /$ ideas.8698

ISSN : 1950-5701

Éditeur

Institut des Amériques

\section{Référence électronique}

Françoise Palleau-Papin, « Le miroir haïtien de Ben Fountain », IdeAs [En ligne], 16 | 2020, mis en ligne le 01 octobre 2020, consulté le 18 octobre 2020. URL : http://journals.openedition.org/ideas/8698 : DOI : https://doi.org/10.4000/ideas.8698

Ce document a été généré automatiquement le 18 octobre 2020

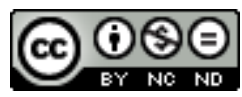

IdeAs - Idées d'Amériques est mis à disposition selon les termes de la licence Creative Commons Attribution - Pas d'Utilisation Commerciale - Pas de Modification 4.0 International. 


\title{
Le miroir haïtien de Ben Fountain
}

\author{
Ben Fountain's Haitian Mirror \\ Espelho haitiano de Ben Fountain \\ Françoise Palleau-Papin
}

\section{Introduction : un changement de perspective}

Cet article vise à examiner la façon dont les nouvelles de Ben Fountain, dans son recueil Brief Encounters with Che Guevara (2006), rendent compte de l'histoire et de la culture haïtiennes et les mettent en fiction dans une perspective non seulement états-unienne, mais américaine. Fountain porte un regard critique sur les O.N.G. étrangères à Haïti et leur action, et cherche à décentrer le regard des lecteurs, plaçant les lecteurs étatsuniens à la marge d'un centre insulaire. Il montre qu'une perspective endogène est la seule qui puisse apporter un renouveau, en ouvrant le regard des pays riches sur les conséquences de leur aide humanitaire ou de leur ingérence en Haïti. Comme l'écrivain parle créole, et connait bien la culture haïtienne, on cherchera à analyser la façon dont il enrichit et «déterritorialise» son anglais (Deleuze, G., 1993: 93), à la fois linguistiquement et idéologiquement, pour regarder les États-Unis d'un point de vue haïtien, plaçant ainsi son propre pays à la marge, et Haïti au centre de sa focale, pour un renouvellement des regards croisés. La fiction de Fountain fait appel au rêve, à l'utopie, à l'art, à un changement de perspective radical pour combattre les dictatures que les personnages de ses nouvelles subissent, et pour changer notre vision du monde. Les personnages étatsuniens de Fountain qui reflètent une position auctoriale (autofictionnelle ou même autobiographique) ne cherchent pas tant à parler de Haïti, ni pour Haïti, qu'à l'entendre de manière personnelle et intériorisée. Ou, pour varier la métaphore de la perception, ils cherchent un miroir qui permette de mettre à distance leur pratique dans la contemplation du reflet, de réfléchir, de faire retour sur soi pour établir un rapport authentique au monde. 


\section{L'« interpersonnalisation »}

2 Les nouvelles de Fountain, même lorsqu'elles ne parlent ni d'Haïti ni du vaudouisme directement, évoquent à plusieurs reprises la dépersonnalisation de la transe vaudoue, par laquelle le sujet en transe n'est plus tant défini selon une individualité qui lui serait propre que traversé par une identité multiple, changeante, devenue véhicule des divinités ou loas. Dans la dernière nouvelle du recueil, une pianiste vivant sous le régime nazi ressent une extase et une dépossession de soi proche du vaudouisme, au point de s'estimer le véhicule d'une transmission artistique qui ne dépend pas d'elle directement : « in a way it's not me, it's something coming through me » (Fountain, B., 2006 : 222). La transmission a ses mystères, d'une culture à l'autre, d'une époque à l'autre, parce que dans les œuvres de Fountain, l'humanité partage des invariants de résistance et de résilience en défiance à tous les totalitarismes. Si la transgression des règles par l'extase vaudoue dans les ouvrages de Fountain a déjà fait l'objet d'une étude (Palleau-Papin 2013), cet article propose ici d'analyser comment les nouvelles de Fountain mettent en œuvre une dépossession de soi allant jusqu'à l'expérience radicale de voir par les yeux de l'autre, à la lumière de la philosophie d'Emmanuel Lévinas.

3 Fountain pousse l'empathie jusqu'à la dépersonnalisation pour laisser place à l'autre, ce qu'on pourrait appeler une « interpersonnalisation ». Plus qu'un vide suggéré par le pré fixe « dé ", il s'agit chez lui d'un échange "inter ", d'un mouvement, par lequel on se laisse habiter par l'autre, ou dans les mots d'un soldat qui estime sans vergogne que la déesse haïtienne de l'amour apparaîtra aussi dans les rêves de sa femme étatsunienne : "We're all connected now.» (63) Dans la nouvelle "The Good Ones Are Already Taken », le soldat de l'armée américaine qui rentre de Haïti explique ainsi à sa femme qu'il a contracté un mariage vaudou avec la déesse de l'amour, Erzulie. Grâce à ce mariage littéralement extraordinaire, sa présence dans les Special Forces de l'armée pour le maintien de la paix prend un tout autre sens. Cette branche des forces armées est entraînée à manipuler les populations locales en s'adaptant à leur culture : «Dirk described his journey into Haitian voodoo, which began as part of the mission, a standard hearts-and-minds tactics of the Special Forces-contact and co-opt the local power structure.» (Fountain, B., 2006: 59) Dépassant la stratégie militaire de la cinquième colonne, l'interpersonnalisation entre en jeu, et pousse le processus plus loin, au-delà de la manipulation culturelle contrôlée, vers l'ouverture totale et la réception sans arrière-pensée. Dirk reçoit plus qu'il n'agit ou ne contrôle, il n'impose pas sa manipulation idéologique à des fins politiques, mais se laisse habiter par une compréhension culturelle dont il ne se soupçonnait pas capable auparavant. À son retour, la transmission de la vision passe à sa femme Melissa, malgré la résistance que celle-ci lui oppose au début. Des mots nouveaux apparaissent alors, pour tenter de dire en anglais la dépossession qui permet de voir par les yeux de l'autre, ou bien des mots anciens sont renouvelés par un usage inattendu. Melissa remarque par exemple que son mari a changé, et qu'elle constate désormais chez lui «a slackening of the male impulse to domineer. » (Fountain, B., 2006: 62) Le verbe « domineer » fait retentir son étymologie latine de maîtrise, et son emploi le plus courant en anglais se limite souvent au participe "domineering ». La forme infinitive, dans le contexte francophone d'Haïti où son mari a séjourné, se rapproche du français «dominer » comme si Melissa avait accès, par son usage de l'anglais, à la pensée de son mari en français, langue de communication avec Erzulie dans les rêves de Dirk. L'essentiel de la rencontre avec le 
vaudouisme tourne ainsi autour du pouvoir étatsunien, considéré comme suprématiste et patriarcal, et du désir de dominer, qui s'estompe chez Dirk au contact de la divinité vaudoue, de même que Dirk a fait l'effort d'apprendre non seulement le français, mais le créole, abandonnant le confort linguistique de sa langue maternelle. Suivant le concept deuleuzien de «déterritorialisation » de la langue maternelle déjà évoqué, la nouvelle met en œuvre une création lexicale qui renouvelle celle-ci. Il ne s'agit pas ici d'un emprunt à une langue autre, mais d'une façon de travailler l'anglais de l'intérieur, de le minorer en une création lexicale singulière.

Un néologisme discret, parce qu'inséré dans le texte sans bouleversement de niveau de langue ni d'habitus linguistique flagrant, révèle en effet le processus qui permet d'accé der à un autre soi dans cette nouvelle. Le substantif «womb» devient un participe passé, « enwombed ", pour dire la prégnance, l'imprégnation humaine d'une idée, un envahissement de l'amour, psychique autant que physique, pour Melissa: «After a while she lost all feeling of the floor, as if she were floating, enwombed in her own sphere of weightlessness» (Fountain, B., 2006: 78). Signe d'une remarquable inventivité linguistique, Fountain renouvelle ici l'anglais courant, par l'ajout d'un pré fixe d'inclusion à un mot d'anatomie, sous l'égide de Shakespeare, grand créateur de mots par l'ajout d'un préfixe (tel le préfixe un- ajouté à un mot existant, selon David Crystal : "There are 314 instances in the Oxford English Dictionary where he is the first citation for an un- usage.» Crystal, D., 2004: 304). Le paradoxe d'une matrice protectrice embrassante (enwomb) qui serait dénuée de poids, comme en apesanteur, la préciosité du participe passé inusité mais compréhensible, tout signale une langue poétique au sens étymologique, qui cherche à décrire un phénomène inconnu en créant un mot pour le dire. Ce préfixe anglais d'origine latine (in-) fonctionne de manière semblable aux agglutinations de deux mots distincts qui sont fréquentes dans le créole haïtien (selon l'étude de Wadner Isidor, on y reviendra), conférant une grande densité à l'expression. Melissa se retrouve ainsi «enwombed», mais pas dans une matrice pesante ni terrestre, puisqu'il s'agit alors pour elle d'habiter une zone en apesanteur, hors de la corporalité tangible, "in her own sphere of weightlessness ", lorsqu'elle d écouvre conjointement qu'il y a du vaudouisme dans sa banlieue rurale :

Was there a homegrown voodoo right under her nose, a french-fried 覧sic Carolina version she'd been missing all this time? It seemed possible as she made her daily commute, staring out from her car past the orderly fields toward the brooding wall of trees in the distance, that deckle-edged veil of luminous green standing in for the less penetrable jungles of the mind.

(Fountain, B., 2006: 75)

Pour Melissa, le paysage luxuriant de la Caroline du Nord participe de sa découverte de l'inconscient et de son enchevêtrement presque impénétrable dans les mailles du conscient, qui est décrit comme une jungle, par déplacement de l'extérieur à une vision intérieure, tellement métaphorisée qu'elle en perd tout ancrage corporel ou même humain. Dans sa matrice en apesanteur et inconsciente, tellement autre qu'elle évoque le monde végétal, Melissa fait passer la voix des autres à travers la sienne, en ventriloque visionnaire.

\section{Décentrer le regard : l'emprunt au créole}

6 La créativité lexicale s'associe chez Fountain à l'emprunt à une langue étrangère pour non seulement décentrer la vision anglophone contemporaine du monde, mais encore, 
pour l'insulariser. L'insularité linguistique et géographique d'Haïti devient alors le centre du monde, la grille de lecture culturelle haïtienne déplaçant l'altérité vers l'anglais du voisin continental. Il est révélateur que la langue étrangère ne soit pas le français standard appris dans les écoles ou l'université où il a étudié le droit, mais le cré ole haïtien, plus proche des amis haïtiens de Fountain, dont Garry (un ophtalmologue que Ben Fountain mentionne dans le podcast " Haiti is Destiny »). La langue vernaculaire est signalée dans les nouvelles en anglais par des emprunts lexicaux qui permettent une mise à distance choisie.

7 La première prise de position personnelle de l'auteur s'affiche dans la nouvelle éponyme, "Brief Encounters With Che Guevara", qui change de contrat narratif, les autres nouvelles étant à la troisième personne, pour introduire une dimension autofictionnelle, voire même autobiographique (bien que seuls de rares entretiens ou articles permettent de vérifier ce contrat autobiographique momentané dans la nouvelle, dont le podcast «Haiti is Destiny ", l'écrivain n'ayant pas à ce jour publié d'autobiographie). La voix narrative passe à la première personne pour dire son investissement personnel, à la fois par l'amitié et par une projection empathique et intellectuelle. Le narrateur dit d'où il parle, mais sans se nommer directement en tant qu'auteur, offrant ainsi une image de sa pudeur ou un effet de flottement de contrat narratif, qui brouille les pistes d'identification de l'auto-fiction.

Chaque section de la nouvelle commence par préciser son âge dans les étapes de sa vie, et dépeint son environnement familial et social. La première section commence ainsi : "When I was six " (Fountain, B., 2006: 183), la deuxième, "When I was twenty " (Fountain, B., $2006: 186$ ), la troisième, « In my early thirties I began making trips to the beleaguered island nation of Haiti.» (Fountain, B., 2006: 193). Il y a un hiatus entre cette première phrase de la troisième section, qui montre certes un intérêt pour Haïti, mais aussi un apitoiement de nanti par la qualification de «beleaguered » qui trahit une vision de l'extérieur, et celle de la quatrième section, qui fait retentir une colère et une mise à distance historique plus radicale, exprimée de l'intérieur cette fois: « Throughout my thirties I kept going to Haiti, convinced that I'd found ground zero for all the stupidity, waste, and horror inflicted on the hemisphere since Colombus and the Spaniards set up shop.» (Fountain, B., 2006 : 196). La référence implicite est celle de la violence du 11 septembre 2001, puis la reconstruction du World Trade Center sur les mêmes bases, à "ground zero". Le phrasé semble impliquer la violence de la colonisation comme source de tous les maux, la base même ("ground zero») de l'horreur infligée aux populations de l'île depuis plus de cinq siècles. À son corps défendant, l'auteur serait implicitement associé à cette violence, par son appartenance nationale à un camp adverse, celui qui a imposé l'embargo, d'où ses visites multiples dans l'île et sa tentative de comprendre personnellement comment les choses en sont arrivées là. La familiarité du ton, avec l'expression "set up shop ", laisse entendre le mercantilisme du colonialisme, qui marque pour beaucoup le début du capitalisme sauvage, celui qui consiste justement à ensauvager et à exploiter, à faire commerce de tout, surtout des humains, mais aussi de la nature à grande échelle. Meurtres et déforestation, prise de pouvoir politique sanguinaire, justification religieuse d'un gé nocide à l'échelle d'un continent, cette phrase légère en incipit de la quatrième section contient des multitudes.

9 Par l'étude de quelques exemples, nous allons tenter de cerner comment le choix des mots haïtiens dépasse le simple effet de couleur locale pour refléter une philosophie. 
Dans son étude de l'alternance de code linguistique, ou code-switching, Penelope Gardner-Chloros distingue l'alternance, le mélange et l'emprunt de la créolisation de contact. Fountain fait retentir l'intraduisible des langues en utilisant l'alternance, mais aussi les convergences, en particulier par le mélange et l'emprunt, entre l'anglais et le créole de la communauté haïtienne. Cette dernière langue, même brièvement incluse dans l'anglais, introduit un point de vue souvent muet dans la littérature étatsunienne, celle de l'anonyme, parfois illettré, qui s'exclame en créole plutôt qu'en français de France, et qui n'a pas voix au chapitre parce qu'il travaille de longues journées, en dehors des lieux de pouvoir.

Citons ici quatre exemples, tirés de la nouvelle "Bouki and the Cocaine", parce que cette nouvelle parle de villageois s'exprimant tous en créole, dans un texte écrit exclusivement en anglais, mais parvenant néanmoins à transmettre une vision haïtienne du monde à travers son intrigue et ses quelques emprunts linguistiques, peu nombreux mais très révélateurs : "réfléchi " (Fountain, B., 2006: 123), «boujwa » (Fountain, B., 2006: 127), «pa-palé » (Fountain, B., $2006: 129)$ et « chadwon» (Fountain, B., 2006 : 130). L'art de la suggestion revient à faire deviner à travers ces quelques mots la parole autochtone, sans qu'aucune phrase créole complète ne soit pourtant transcrite dans l'anglais de cette nouvelle (contrairement à celle intitulée « Rêve Haï tien ", par exemple, qui est plus évidente dans sa citation d'une phrase créole entière). Un pêcheur très pauvre, Syto Charles, détourne plusieurs cargaisons de cocaïne, et face à la corruption des autorités, décide de faire profiter tout son village du gain en se servant du carnaval comme d'une diversion pour transporter la drogue à la capitale, cousue dans les costumes des habitants du village habillés en gédés pour l'occasion. Cette nouvelle d'une grande richesse met en œuvre une philosophie de l'union des plus modestes, qui s'appuient sur leur tradition culturelle pour déjouer la corruption mafieuse de leurs élites, qui préfèrent l'enrichissement personnel au bien commun, et la menace des trafiquants internationaux entre l'Amérique du Sud et du Nord. Le personnage principal, Syto, porte dans le titre de la nouvelle le surnom emblématique de Bouki, qui est un "personnage des contes Cric? Crac! de Georges Sylvain», et signifie de manière caricaturale un «sot, idiot, imbécile (naïf)» (Isidor, W., 2016 : 545). Dans son recueil publié en 1901, Georges Sylvain annonce dans son sous-titre une version acclimatée des «Fables de La Fontaine racontées par un montagnard haitien et transcrites en vers créoles.» Son personnage légendaire de Bouki est ici repris par Fountain, qui inscrit son titre dans la tradition des contes créoles populaires. Chez Fountain, Syto est un Bouki contemporain plus complexe que celui de la fable, puisqu'il se sert justement de ce nom générique comme d'un déguisement pour jouer les benêts afin de tromper les trafiquants et la police corrompue. L'hommage à Sylvain illustre une politique de l'adaptation culturelle par l'écriture. De même que Sylvain a, selon l'expression de Kathleen Gyssels dans sa présentation du volume, "haïtianisé » les fables de La Fontaine (Gyssels, K., 2011 : Xv), Fountain à son tour américanise les contes haïtiens de Sylvain dans son recueil, pour à la fois les rendre plus proches de ses lecteurs étatsuniens et en retour, amener ceux-ci à une autre vision du monde hors de leurs frontières.

11 En contexte dans la nouvelle, chaque mot créole emprunté est clair, bien que livré le plus souvent sans glose explicative, pour plonger plus immédiatement dans une vision locale de la diégèse. En ce qui concerne le "chadwon », le texte laisse deviner qu'il s 'agit d'un oursin («sea urchin» Fountain, B., 2006: 130), plutôt que l'argémone mexicaine, ou faux chardon du Mexique. Libre à nous de faire le rapprochement avec le 
chardon pour ses piquants. Ce qui importe ici, c'est de faire retentir le mot créole dans son étrangeté à l'anglais, et au français dans une moindre mesure, pour le plaisir du dépaysement. Dans son édition de contes créoles antillais, Patrick Chamoiseau insiste quant à lui sur le mystère des mots empruntés au créole et livrés sans glossaire au lecteur de français : «Laissez faire, dessous les mots étrangers, la magie souterraine » (Chamoiseau, P., 2018: 16). Par l'emprunt, le dépaysement opère, magiquement, jusqu'au cœur des lecteurs.

12 Fountain cherche lui aussi à toucher au plus juste d'un affect dans les mots mêmes de la souffrance non traduite. Lorsque le narrateur précise que l'épouse du héros Syto est frappée de mutisme, ne pouvant surmonter son deuil de leur fille unique morte par manque de médicaments sous l'embargo, c'est le mot créole qui fait retentir la force de cette privation de parole : "She never spoke-she'd had the pa-palé disease ever since their daughter's death-but it wasn't a surly or raging silence, Syto knew that now. » (Fountain, B., 2006 : 129) Pour dire le mutisme, le mot français parlé est en créole haï tien agglutiné d'une négation, pa-palé (Isidor, W., 2016 : 71) et prend plus d'importance par sa densité de mot agglutiné et par son étrangeté dans la phrase en anglais, mise en relief par les italiques, faisant ainsi retentir le silence des paysans pauvres qui subissent la corruption de leurs dirigeants, un sujet central de la nouvelle et du recueil. Les habitants du village restent muets et se gardent bien de protester lorsqu'ils sont harcelés par les survols d'hélicoptères des forces de l'armée américaine la nuit et par les intimidations des hommes de main du régime en place, qui sillonnent la campagne dans leurs pickups montés d'armes de guerre. Le mot français ou créole permet de revenir sur ce qui a été dit, sur ce qui achoppe, quand l'anglais ne suffit pas à dire les choses. Ainsi, le mot "réfléchi» vient préciser ultérieurement ce que l'anglais ne saisissait pas complètement, lorsque Syto se pose tant de questions sur son sort et celui des siens :

Tonight he baited his string of hooks with pisket and chicken guts, spotlit the water with his lampe-batterie, and then drifted, not so much thinking about things as biding with a certain awareness of his life. Réfléchi, that was better than direct thinking for the world of problems you could never really solve. The problem of contraband, for example, or the confusion of politics, or the trouble that came of needing to eat every day. Or the death of children, a cruelly regular thing in Trois Pins.

(Fountain, B., $2006: 123$, c'est l'auteur qui souligne)

Le mot français, qui a donné «reflect» en anglais, parle d'indirection de la lumière, lorsqu'une surface renvoie l'éclairage, la diffraction le rendant indirect. Il s'agit pour Syto d'une manière de penser qui se traduit en manière d'agir dans la nouvelle. Il ne peut lutter de front, mais trouve une manière insidieuse de résister à la corruption. Sa pensée devient aussi l'image de la stratégie narrative à l'œuvre dans la nouvelle, qui ne laisse comprendre la manœuvre qu'après coup, indirectement. Le passage met en italique le pisket, les alevins séchés très utilisés dans la cuisine haïtienne, parce que c'est un produit local dont l'anglais ou le français ne peuvent approcher que par une glose plus ou moins explicative dans la traduction. Le mot "lampe-batterie ", facile à deviner pour un lecteur anglophone, rend compte d'une réalité rurale, qui est le manque d'électrification des zones isolées. Le mot "réfléchi» vient expliquer la tentative de description de la pensée, plus proche de l'attentisme ; le mot « biding » fait appel à des syntagmes figés comme « biding his time », tandis que la réflexivité de toute pensée, de tout retour sur l'expérience, de toute conceptualisation de l'immanence, est circonstanciée par le complément "with a certain awareness of his life ». En résumé, 
voilà ce qui est « réfléchi » et va se déployer dans l'espace de la nouvelle en véritable stratégie narrative : un mode d'être au monde qui, indirectement mais efficacement, critique la corruption en place, locale comme internationale, en donnant le point de vue local plutôt que celui des O.N.G. internationales, par la mise en relief d'emprunts au créole haïtien dans le texte en anglais.

La nouvelle met ainsi en scène un rapport à l'argent qui pose problème lorsqu'il se rapproche de la conception ultra-capitaliste des trafiquants de drogue ou des autorités qu'ils achètent. Syto s'inquiète de la réaction de son frère Lulu, lui-même tenté par le gain personnel, avant que Syto n'invente une solution collective pour le village entier : " For two weeks Lulu didn't miss a day on the boat, and he talked about money so incessantly that Syto wondered if his brother had gone boujwa, money-crazy.» (Fountain, B., 2006: 127) Le mot créole ainsi traduit en anglais prend une force bien plus grande que le français «bourgeois» qu'il laisse encore entendre. L'embourgeoisement n'est pas ici un résultat, le fait d'être un nanti, mais un processus mental, celui d'être motivé uniquement par l'appât du gain. Si la possibilité d'un enrichissement est très alléchante pour les villageois appauvris par l'embargo, au point de constituer une obsession pour Lulu, elle risque de les pervertir. La fille de Syto étant morte par manque de médicaments pendant l'embargo, Syto Charles aurait lui aussi toutes les raisons d'être tenté par la même obsession que son frère, et pourrait penser à lui-même avant tout autre. Mais il comprend que devenir boujwa mène à tout, à l'individualisme forcené ou au meurtre, par une forme d'égarement allant jusqu'à la folie («money-crazy »), ce qu'il refuse obstinément, fidèle à son éthique des petits contre les forts, du collectif contre l'individuel.

\section{L'argent comme miroir déformant}

Au cours de ses voyages en Haïti depuis 1991 (Fountain, B., 2013), Fountain comprend quant à lui que la critique majeure que les Haïtiens font des Nord-Américains est celle de leur rapport à l'argent, ce qu'il traduit dans ses personnages clés, véhicules d'une philosophie auctoriale que l'on peut cerner dans ses articles sur les élections présidentielles américaines de 2016, publiés dans The Guardian, puis rassemblés dans son ouvrage Beautiful Country Burn Again). Il n'a de cesse d'éduquer ses contemporains à l'histoire d'Haïti et à une vision culturelle qu'il partage dans ses articles dont l'un explique son intention politique dans ses nouvelles : «In one way or another, all of the stories in Brief Encounters with Che Guevara deal with matters of power and profit, usually in what might be called the world's "hot zones" - Haiti, Burma, Colombia, Sierra Leone. But Haiti is the place I return to time and again. » (Fountain, B., 2013)

Dans la nouvelle "Rêve Haïtien", un résistant haïtien s'impatiente et tance le personnage focalisateur :

You are American, so of course everything for you is a question of money. Honor and courage count for nothing, justice, fear-those people in the palace are cowards, okay? When the real fighting starts I assure you they will run. They will pack their blood money in their valises and run.

(Fountain, B., $2006: 39$ )

17 Fountain revient sur l'importance de l'argent et du financement des campagnes électorales dans son analyse de la civilisation étasunienne Beautiful Country Burn Again; 
il y cite l'ouvrage de Barack Obama, The Audacity of Hope, dans lequel Obama revient sur la conséquence de sa recherche de fonds :

I know that as a consequence of my fund-raising I became more like the wealthy donors I met. I spent more and more of my time above the fray, outside the world of immediate hunger, disappointment, fear, irrationality, and frequent hardship of ... the people that I'd entered public life to serve.

(Cité par Fountain, B., 2018: 32)

Les électeurs ressentent ce gauchissement du regard, lorsque le contact direct s'écarte du quotidien des invisibles, ceux qui n'ont pas accès aux lobbys et ne vont pas aux pinces-fesses de Goldman Sachs, contrairement à Hillary Clinton. Fountain spécule ainsi sur les conséquences de cette perception :

Obama brought a crucial measure of self-awareness to the money hustle, a capacity to detach and evaluate himself with a critical eye. The moral capacity, we could call it. It didn't stop him from scooping up wheelbarrows full of campaign money, but his ability to form and articulate a morally ambitious politics-one that had room for self-doubt-was surely part of why voters responded so strongly to him, at Hillary's expense.

(Fountain, B., $2018: 33$ )

En passant par Haïti, le regard se retourne ainsi vers la politique étatsunienne. Le miroir sombre d'une île martyre rend visible une faillite humaine à l'échelle d'un payscontinent, celle du gain comme mesure de la valeur parce qu'il manque totalement d'interpersonnalisation, et empêche de voir par les yeux d'autrui, ainsi relégué à une altérité irréductible.

\section{De la tradition afro-américaine au « penser-à-l'autre »}

Par sa décentralisation du regard, Fountain s'inscrit dans une tradition afro-américaine qu'il revendique dans son article sur Haïti écrit pour The Guardian. Il y mentionne un discours de Frederick Douglass prononcé à Chicago 1893, en le contextualisant :

Despairing that slavery would ever be abolished in the US, Douglass and his family were preparing to relocate to Haiti when word came of Lincoln's signing of the Emancipation Proclamation. Douglass decided to remain in the US, and later served as the country's ambassador to Haiti. In its analysis of Haiti's role in world history, of the manipulations of foreign powers and business interests in its internal affairs, and of its future prospects, Douglass's lecture is so prescient that it might have been written last week.

(Fountain, B., 2013)

21 Il s'appuie sur la réflexion critique de Douglass sur son pays à partir de Haïti et des relations de l'île nation avec les États-Unis. Douglass insistait sur la mise en miroir des deux pays, en préambule de son discours :

There are many reasons why a good understanding should exist between Haiti and the United States. Her proximity; her similar government and her large and increasing commerce with us, should alone make us deeply interested in her welfare, her history, her progress and her possible destiny.

(Douglass, F., $1893: 3$ )

Zora Neale Hurston avait bien compris elle aussi que la mise en miroir des deux pays fascine parce que d'autres perspectives pour les anciens esclaves et d'autres relations entre les communautés paraissent alors possibles dans les états du Sud comme la Floride ou la Louisiane. Avant d'aller en Haïti, Hurston s'était rendue à la Nouvelle- 
Orléans pour étudier le vaudouisme, qu'elle appelle dans son autobiographie «sympathetic magic " (Hurston, Z., 1984 : 191). La magie de la sympathie consisterait chez Hurston en un don de double vue grâce aux pouvoirs du loa. Fountain élargit la source du don à la capacité de voir par les yeux de l'autre, au point de penser pouvoir agir sur l'autre à distance, les "médecins à deux têtes " étant alors capables de se projeter dans la tête d'autrui (et les dédoublements étaient multiples aussi dans l'expression de Hurston: «In order to work with these 'two-headed' doctors, I had to go through an initiation with each. » Hurston, Z., 1984 : 191).

Dans la nouvelle "The Good Ones are Already Taken", l'épouse du soldat qui a contracté un mariage avec la déesse vaudoue Erzulie cherche à comprendre son mari Dirk. Son désarroi mène Melissa à reprendre contact avec une cousine éloignée, Rhee, qui est "psychic» de profession, cette voyante ou médium pratiquant l'art de se projeter dans le psychisme d'autrui. Dès le premier appel téléphonique, Melissa a une intuition de la capacité de vision paranormale de Rhee, que rien ne surprend : « Melissa resisted the thought that Rhee had been expecting her call.» (Fountain, B., 2006: 71) Rhee ressent les «ondes» qui ressemblent à de la prémonition, ce qu'elle appelle communément "some funny vibes on this ", à moins que ce ne soit qu'une perception accrue d'un réel invisible (Fountain, B., 2006: 76) et pour mieux ressentir les choses, elle demande à voir l'autel que Dirk a érigé à la déesse vaudoue, dans le trailer du couple. La reprise de contact entre les cousines, la capacité de projection de Rhee dans l'univers du couple, l'union des « vibrations » invisibles des psychismes entre Haïti et la Caroline du Nord, voilà en action le résultat de ce que Dirk proclame avec assurance depuis son retour, comme on l'a vu: «We're all connected now.» (Fountain, B., 2006: 63) Ces échanges vont au-delà d'un simple dépassement de l'individualisme, ce ne sont pas seulement des marques de sociabilité mais une communication qui engage tout l'être, jusqu'à l'intimité du psychisme.

De tels échanges ne sont pas sans conséquences politiques profondes. Plonger dans la "magie sympathique», comme dit Hurston, ou dans l'échange vibratoire de « connections » invisibles mais puissantes, selon le personnage Dirk, tout conduit les héros de la nouvelle à regarder leur pays avec une nouvelle compréhension, élargie à celle des autres, y compris ceux qu'on avait envahis. Melissa conclut un paragraphe d'échange avec sa cousine Rhee par le constat d'une évidence, soudainement claire à ses yeux : "They hung up, and Melissa decided that she didn't feel crazy. It seemed, rather, that reality itself had gone mad, and she was riding her own little scrap of sanity through the cosmic whirlwind. ( (Fountain, B., 2006: 76) On retrouve dans cette phrase toute la réflexion éthique que Fountain met en fiction dans son roman sur la guerre d'Irak, Billy Lynn's Long Halftime Walk (Fountain, B., 2012). Quand c'est le pays qui est fou, les soldats qui souffrent de traumatismes sont les plus normaux, en particulier quand les troupes sont exhibées comme autant de trophées à l'occasion d'un grand match de football, avec toute l'obscénité d'un monde où les échanges humains sont corrompus par l'argent et le mercantilisme que les soldats refusent.

Dans la nouvelle, le contact renouvelé entre Dirk, Melissa et Rhee permet de mettre en œuvre par la fiction ce qu'Emmanuel Lévinas appelle «l'entre nous» au sens profond $\mathrm{du}$ «penser-à-l'autre ». On ne peut guère penser à soi tout seul sans conscience de l'autre, parce que selon Lévinas, « la pensée commence avec la possibilité de concevoir une liberté extérieure à la mienne », ce qu'il exprime dans sa belle formule d'un regard porté au visage d'autrui comme du monde : «Le monde de la perception manifeste un 
visage : les choses nous affectent comme possédées par autrui. » (Lévinas, E., 1991: 27, c 'est l'auteur qui souligne). Lévinas ne parle certes pas de la transe vaudoue dans sa vision philosophique, mais la possession que met en fiction Fountain dans ses nouvelles participe de l'échange de regard et de la mise en miroir d'un monde qui n'est jamais seulement propre à soi ni propriété privée, mais toujours partagé avec autrui. Fountain construit un monde aux solidarités renouvelées dans le présent de l'échange. "We're all connected now. » (Fountain, B., $2006: 63$ )

\section{Conclusion : la mémoire du parcours}

Les nouvelles de Fountain opèrent un décentrement, à travers l'itinéraire d'une écriture qui fait le choix d'écrire un livre américain par le détour d'Haïti. Dans sa suspension du jugement, dans sa tentative de voir par les yeux de l'autre et de se mettre à distance salutaire des préjugés de l'éducation, Fountain est sensible à la $r$ éflexion, au sens de pensée, que le reflet indirect du miroir renvoie à son pays, en particulier en tant qu'habitant de Houston. Ses nouvelles invitent à explorer un sens du collectif accru, moins individualiste que dans sa culture, et par là même, une autre relation à l'argent, signe de corruption égoïste perceptible dans la réussite individuelle. Elles invitent aussi à une autre conscience du passé, plus présent en Haïti et en Louisiane à travers le carnaval et la fête des morts. Le collectif s'enrichit d'une épaisseur temporelle et interpersonnelle, rendue présente de manière spectaculaire dans les épisodes de transe vaudoue, et sous-jacente le reste du temps. En déplaçant ces moments de transe jusqu'en Caroline du Nord, en ironisant en passant sur les cultes chrétiens protestants (des congrégations Cavalry Baptist et First Methodist, Fountain, B., $2006: 65$ ), Fountain ouvre ainsi la possibilité d'une spiritualité et d'une politique d'un autre ordre. Sa vision inclut celle d'Haïti au terme d'un parcours centrifuge qui fait retour sur soi. De la fuite du centre au retour centripète, ce qui compte dans le déplacement, c'est de garder la mémoire du parcours. La vision ainsi renouvelée est davantage tournée vers une communication conjointe entre les humains, le divin et les ancêtres, dans une communauté étendue, dépersonnalisée de son individualisme et magiquement, idéalement, interpersonnalisée.

\section{BIBLIOGRAPHIE}

Chamoiseau, Patrick, « Préface, les contes de la survie », Contes des sages créoles, Paris, Seuil, 2018, p. 11-16.

Crystal, David, The Stories of English, London, Allen Lane, 2004.

Deleuze, Gilles, Critique et Clinique, Paris, Éditions de Minuit, 1993.

Douglass, Frederick, « Lecture on Haiti », The World's Fair, Jackson Park, Chicago, Jan. 2nd, 1893, http://faculty.webster.edu/corbetre/haiti/history/1844-1915/douglass.htm, page consultée le 10 septembre 2019. 
Fountain, Ben, Brief Encounters With Che Guevara, New York, Harper Perennial, 2007 [2006].

Fountain, Ben, Billy Lynn's Long Halftime Walk, New York, Ecco/HarperCollinsPublishers, 2012.

Fountain, Ben, Beautiful Country Burn Again. Trump's Rise to Power, and the State of the Country that Voted for Him, New York, HarperCollins Publishers, 2018.

Fountain, Ben, « Haiti is Destiny », 21 mai 2010, https://www.thisamericanlife.org/408/islandtime/act-three-0, page consultée le 14 février 2020.

Fountain, Ben, « Ben Fountain's top 10 books about Haiti », 15 mai 2013, https://

www.theguardian.com/books/2013/may/15/ben-fountain-top-10-haiti-books, page consultée le 29 mai 2020.

Gardner-Chloros, Penelope, Code-Switching, Cambridge, Cambridge University Press, 2009.

Gyssels, Kathleen, « Introduction : La Fontaine, relu par Georges Sylvain », Georges Sylvain, Cric? crac! Fables de La Fontaine racontées par un montagnard haïtien et transcrites en vers créoles, Paris, L'Harmattan, 2011, p. VII-XXXIII.

Hurston, Zora Neale, Dust Tracks on a Road, New York, Harper \& Row, 1984 [1942].

Isidor, Wadner, Petit lexique du créole haïtien : vocabulaire, Longueuil, Québec, Les Éditions Connaivie, 2016, édition Kindle.

Lévinas, Emmanuel, Entre-nous. Essais sur le penser-à-l'autre, Paris, Grasset, 1991.

Palleau-Papin, Françoise, « "Pitch the king” : Ben Fountain's games », Transatlantica, 2, 2013, 30 avril 2014, http://journals.openedition.org/transatlantica/6596, page consultée le 30 avril 2019.

Sylvain, Georges, Cric ? crac ! Fables de La Fontaine racontées par un montagnard haïtien et transcrites en vers créoles, Paris : L’Harmattan, 2011 [1901]

\section{RÉSUMÉS}

Cet article étudie la manière dont les nouvelles de Ben Fountain mettent en place une « interpersonnalisation », par le biais de l'emprunt linguistique au créole haïtien entre autres stratégies narratives, pour mettre en relation les personnages, à l'instar de la transe vaudoue qui dépersonnalise. La mise en miroir entre les États-Unis et Haïti permet à Fountain de réfléchir à la relation que les personnages entretiennent entre eux et avec l'argent, et à envisager de nouvelles formes d'échange et façons d'être au monde, à la manière du «penser-à-l'autre » de la philosophie d'Emmanuel Lévinas.

This paper examines how Ben Fountain's short stories depict interpersonal relationships between the characters, not unlike voodoo possession which depersonalizes, borrowing from Haitian Creole amongst other narrative means. Setting up Haiti as a mirror to the United States, Fountain examines how the characters relate to one another and to money, and imagines new forms of exchange and new ways of being in the world, "Thinking-of-the-Other" as in the philosophy of Emmanuel Lévinas.

Este artigo estuda a maneira como os contos de Ben Fountain estabelecem uma "interpessoaliza ção", através de empréstimos linguísticos do crioulo haitiano, entre outras estratégias narrativas, para relacionar os personagens, a exemplo da transe do vodu que despersonaliza. 0 espelhamento entre os Estados Unidos e o Haiti permite que Fountain reflita sobre o relacionamento que os personagens mantêm entre si e com o dinheiro, e considere novas formas de trocas e de estar no mundo, à maneira de "pensar o outro" da filosofia de Emmanuel Lévinas. 
INDEX

Mots-clés : Interpersonnalisation, « penser-à-l'autre », vaudouisme, miroir, créole haïtien

Palavras-chave : interpersonalização, « pensar o outro », vodu, espelho, Crioulo haitiano.

Keywords : Interpersonalization, “Thinking-of-the-Other”, voodoo, mirror, Haitian Creole

\section{AUTEUR}

\section{FRANÇOISE PALLEAU-PAPIN}

Françoise Palleau-Papin est professeur de littérature américaine à l'Université Sorbonne Paris Nord. Elle est l'auteur d'une monographie critique sur David Markson (This Is Not a Tragedy, Dalkey Archive, 2011) et d'une étude d'un roman de Willa Cather, My Antonia (Atlande, 2016). Elle a dirigé un ouvrage critique sur le roman The Rifles de William T. Vollmann (Under Fire, Peter Lang, 2016) et écrit de nombreux articles sur des écrivains contemporains, dont Ron Rash, Carole Maso, Ben Fountain, Karen Tei Yamashita, Sesshu Foster, Vanessa Place, Anne Carson, Hernan Diaz et Alexis Wright. 